\title{
Research on the ultraviolet laser of LBO crystal
}

\section{Feng Hui Cao ${ }^{1, a}$,Yu Cao ${ }^{2, b}$, Yu Chun $\mathrm{Li}^{1, \mathrm{c}}$ Xiu Ming $\mathrm{Li}^{1, \mathrm{~d}}$}

${ }^{1}$ School Of Mechatronic Engineering, Daqing Normal University, Daqing 163712, China

${ }^{2}$ Changchun New Industry Optoelectric Technology Co., Ltd, Changchun130103, China

acaofenghui@126.com, ${ }^{b} 287716562 @ q q . c o m,{ }^{c}$ liyuchun7@sina.com, ${ }^{d}$ lixiuming4518@163.com

\section{Keywords: laser; Frequency multiplication technology ;LBO crystal}

\begin{abstract}
At first, this paper has summarized the theory of frequency doubling crystal, summarized suitable for uv frequency doubling of the characteristics of nonlinear optical crystal, put forward the theory basis of frequency doubling crystal KTP crystal, debug out of efficient cavity frequency doubling green laser. Choose the LBO crystal, designed an ultraviolet laser, stability of $25.7 \mathrm{mw}$ $355 \mathrm{~nm}$ uv laser output, the conversion efficiency is $11.3 \%$.
\end{abstract}

\section{Introduction}

In recent years, laser technology is more advanced countries in the world, such as America, Britain, Japan, France, Germany, set off a boom in the development of the ultraviolet laser, thanks to its broad prospect of application and industrialization of potentially huge market value. Uv laser by short wavelengths, energy is more concentrated, higher resolution, the ultraviolet laser as a new development direction of the laser. Nonlinear optical frequency conversion technology has been a hot research topic in the field of laser technology. Today can be a variety of frequency is obtained by frequency conversion device of high power laser, also can from the infrared to the ultraviolet wavelength range. The invention of the latest high quality nonlinear optical crystals, nonlinear optical frequency conversion technology is widely used, but also make a branch of the door has become the most active in nonlinear optical fields, one of the most mature branch.

\section{Summary of frequency multiplication principle}

Since the laser appears, it is enough to reflect light material nonlinear response of the light field. This related to the intensity of optical effect, often referred to as nonlinear optical effect. In only as a description of it, will be launched for the nonlinear optical medium CLP polarization P outside light field E form of power series, namely

$$
\vec{P}=\varepsilon_{0}\left[\chi^{(1)} \vec{E}+\chi^{(2)} \vec{E} \vec{E}+\chi^{(3)} \vec{E} \vec{E} \vec{E}+\cdots \cdots \cdot\right]
$$

Consider medium under quadratic nonlinear electric polarization effect, if there are two columns of different frequency of plane wave in the crystal reached the match in place, set for the $\mathrm{Z}$ axis direction,

$$
\begin{aligned}
& E_{1}(Z, T)=E_{1} \cos \left(\omega_{1} t-K_{1} Z\right) \\
& E_{2}(Z, T)=E_{2} \cos \left(\omega_{2} t-K_{2} Z\right) \\
& P=\varepsilon_{0} \chi^{(1)}\left[E_{1}(Z, T)+E_{2}(Z, T)\right]+\varepsilon_{0} \chi^{(2)}\left[E_{1}(Z, T)+E_{2}(Z, T)\right]^{2}
\end{aligned}
$$

Polarized wave not only contains the fundamental frequency $\omega_{1} 、 \omega_{2}$, omega, omega also appear frequency doubling, and frequency and difference frequency, obviously if the incident two columns of wave frequency for $\omega_{1}$ and $2 \omega_{1}$ omega, omega and the frequency of omega $3 \omega_{1}$, that is three times the frequency. By the same frequency multiplication technology can converts the frequency of omega $\omega_{1}$ laser frequency to $2 \omega_{1}$ green, omega travel through the frequency doubling frequency can 
be obtained as 4 omega $4 \omega_{1}$ deep ultraviolet laser.

\subsection{Biaxial crystals in three waves interaction phase matching}

The refractive index of biaxial crystal surface is four surface in rectangular coordinate system (double shell side), the lack of symmetry, the phase matching curve is not a simple analytic solution. Fundamental frequency phase velocity of light in the crystal phase velocity equal to the second harmonic, fundamental frequency light through the crystal, the direction of propagation of excitation frequency doubling polarization field because of the same phase and reinforce each other, so as to achieve the goal of phase matching[1].

According to the polarization direction of incident base wave, phase matching method of can be divided into two categories: the first class I and class II. Such as the incident wave are slow light, polarization direction parallel, called type I phase matching; Such as incident wave both slow and fast light, orthogonal polarization direction, it is called a type II phase matching. The same as the uniaxial crystal, phase matching problem, can turn ratio for the refractive index of the intersecting line problem. As a result of the fast and slow light of biaxial crystal refractive index, respectively for the refractive index of the inner shell surface and the outer shell surface, so I kind of match is composed of fundamental wave slow light and fast light refractive index of the space of the harmonic line, namely by the fundamental harmonic of inner shell surface and exempt from the line[2]. Class II phase matching is fundamental wave refraction surface inside and outside layer on the surface of the shell and the average shell surface and the harmonic on the surface of the inner space. The phase matching condition of electric field can be expressed as[3]:

$$
\begin{array}{ll}
\text { I } & \vec{E}\left(\omega_{1}, n_{1}^{\prime}\right)+\vec{E}\left(\omega_{2}, n_{2}^{\prime}\right) \rightarrow \vec{E}\left(\omega_{3}, n_{3}^{\prime \prime}\right) \\
\text { II } & \vec{E}\left(\omega_{1}, n_{1}^{\prime}\right)+\vec{E}\left(\omega_{2}, n_{2}^{\prime \prime}\right) \rightarrow \vec{E}\left(\omega_{3}, n_{3}^{\prime \prime}\right)
\end{array}
$$

\subsection{And the theory of frequency}

Three waves interaction steady coupled wave equations. Three wave complex amplitude is :

$$
\begin{aligned}
& A_{1}(z)=E_{1}(z) \exp \left[i \varphi_{1}\right] \\
& A_{2}(z)=E_{2}(z) \exp \left[i \varphi_{2}\right] \\
& A_{3}(z)=E_{3}(z) \exp \left[i \varphi_{3}\right]
\end{aligned}
$$

Three collinear phase matching, and the introduction of slowly varying amplitude approximation

$$
\left|\frac{\partial^{2} A_{n}}{\partial z^{2}}\right| \ll\left|K_{n} \frac{\partial A_{n}}{\partial z}\right| \ll\left|K_{n}^{2} A_{n}\right|, n=1,2,3
$$

Eventually available coupled wave equation:

$$
\begin{aligned}
& \frac{d A_{1}}{d z}=i B_{1} A_{3} A_{2}^{*} \exp [-i \Delta k z] \\
& \frac{d A_{2}}{d z}=i B_{2} A_{3} A_{1}^{*} \exp [-i \Delta k z] \\
& \frac{d A_{3}}{d z}=i B_{3} A_{3} A_{2}^{*} \exp [i \Delta k z]
\end{aligned}
$$

\section{The nonlinear characteristics of frequency doubling crystal}

Oxygen titanium potassium phosphate crystals $\left(\mathrm{KTiOPO}_{4}\right) \mathrm{KTP}$ crystals, is negative biaxial crystal, the lattice structure of orthogonal crystal system, density $2.945 \mathrm{~g} / \mathrm{cm}^{3}$, pervious to light band is $350-4500 \mathrm{~nm}$, damage threshold of $300-500 \mathrm{MW} / \mathrm{cm}^{2}$, has high nonlinear coefficient (about 15 times of KDP), high heat conductivity (2 times of BNN crystals), moisture absorption and deliquescence, without separation under $900^{\circ} \mathrm{C}$, good mechanical properties and crystal surface polishing, easily mismatch degree is small. 
KTP crystal when he worked in short wavelength absorption coefficient is greater than the long wavelength, so when running under the condition of short wavelength, pay attention to the heat dissipation of KTP crystal, in order to avoid thermal damage. KTP crystal frequency doubling of the shortest wavelength of $994 \mathrm{~nm}$, harmonic is $497 \mathrm{~nm}$, wavelength less than $994 \mathrm{~nm}$, matching points, there is no class II and class I matching the nonlinear coefficient is far less than class II, so I basic don't match[4].

Because the KTP crystal has high nonlinear coefficient, pervious to light band width, by widespread application Yu Beipin, and frequency, and in the process of optical parametric, especially suitable for $\mathrm{Nd}^{3+}$ :YAG laser (wavelength $1064 \mathrm{~nm}$ ) as The Times of experiments[5].

Three lithium borate crystals $\left(\mathrm{LiB}_{3} \mathrm{O}_{5}\right)$ LBO crystal[6], is a good high-power ultraviolet frequency doubling crystal, have wide at the same time allow Angle and small off the Angle, has been successfully used in YAG, YLF and YAP laser double and triple frequency, belong to the negative biaxial crystal, the crystal structure of orthorhombic system, melting point at $834^{\circ} \mathrm{C}$, density $2.47 \mathrm{~g} / \mathrm{cm}^{3}$, pervious to light with wide band, pervious to light cover a wide range of 160-2600 nm, effective nonlinear coefficient (SHG) which is about three times that of KDP. Three lithium borate crystal has a wide scope of class I and class II non-critical phase matching, so the critical phase matching can be adopted to overcome the walked away from the effect to obtain high frequency doubling efficiency, and at the same time by the dispersion equation, we can calculate its temperature matching features[7] .

\section{Experimental apparatus and conclusions}

\subsection{Lamp pumped electro-optic $Q$ green laser}

We adopted the xenon lamp pumped electro-optic Q produced $1064 \mathrm{~nm}$ pulse output, outside the cavity using KTP crystal frequency doubling, because of the type I phase matching nonlinear coefficient is very small, generally do not use, so we adopt class II phase matching. For type II phase matching requires fundamental frequency light polarization direction orthogonal to each other, thus, when two mutually orthogonal component numerically equal to the fundamental wave, within the crystal frequency doubling efficiency is highest. Therefore, experiment, KTP crystal 45 o Angle placed, to achieve maximum efficiency of frequency doubling. In the experiment KTP crystal transformation frequency of $49.1 \%$.

\subsection{Outside the cavity frequency doubling $\mathrm{Nd}^{3+}$ : YAG/KTP/LBO ultraviolet laser}

We use the $\mathrm{Nd}^{3+}$ :YAG outside the cavity is three times the frequency of $355 \mathrm{~nm}$ uv laser, in order to obtain high quality output mode, working temperature control in $2210 \mathrm{c}, \mathrm{Nd}^{3+}$ : YAG laser using flat - a flat chamber design, cavity length is $0.38 \mathrm{~m}$. Placed in KTP crystal ahead of LBO crystal size, through the dispersion prism $1064 \mathrm{~nm}, 532 \mathrm{~nm}$ and $355 \mathrm{~nm}$ three separate wavelengths of light, the output power is measured with a power meter.

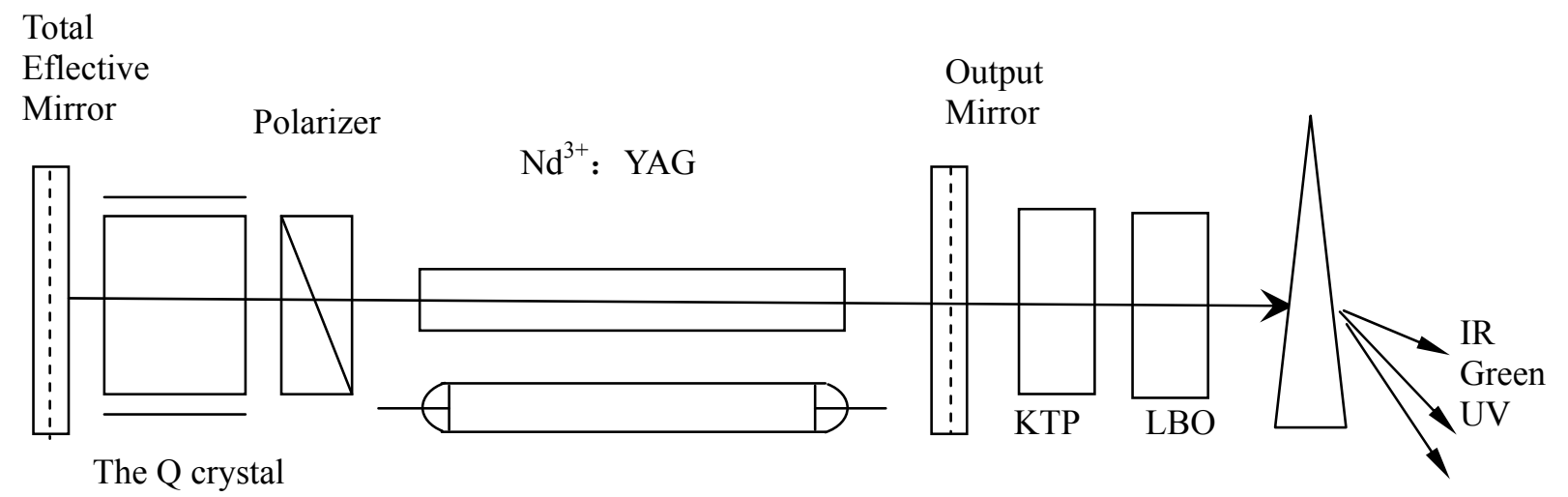

Figure 1 experiment device structure 
Due to wave and frequency have benefited from the fundamental frequency and second harmonic in the nonlinear crystal internal effective energy transformation, and transformation process must meet the energy and momentum conservation at the same time, so you have to consider the relationship between the various wave polarization, also is the placement of LBO crystal Angle. Is twice the frequency acquired using the KTP crystal type II phase matching (), make the fast axis and of KTP crystal $\mathrm{Nd}^{3+}$ : YAG crystal optical axis is 450 , by the same token, placed parallel to the KTP crystal LBO crystals.

Known from the analysis of the above, should be carefully adjust the placement of LBO Angle to the highest harmonic conversion efficiency. Through the experiment, when the working voltage $840 \mathrm{v}$, the stability of $25.7 \mathrm{mw} 355 \mathrm{~nm}$ uv laser output, the conversion efficiency is $11.3 \%$.

\section{Conclusion}

This paper first describes the theory of frequency doubling crystal, summarized suitable for uv frequency doubling of the characteristics of nonlinear optical crystal, put forward the theory basis of frequency doubling crystal KTP crystal, LBO crystal design $\mathrm{Nd}^{3+}$ : YAG laser, uv stability of 355 $\mathrm{nm}$ uv laser output, the conversion efficiency is $11.3 \%$.

\section{Acknowledgements}

This work is financially supported by daqing guidance of science and technology plan projects (szdfy-2015-59) and daqing normal university natural science foundation of China (12ZR14).

\section{References}

[1] Xiao yu zhang, Hong tao gao, Chang tao wang, Chong xi zhou, Chun lei du,Ting wen xing, Han min yao. Shock wave modulation photonic crystal frequency conversion effect research [J]. Journal of electrical engineering, 2008, (3).

[2] Tao jiang. The principle and application of nonlinear optical glass [J]. Laser and optoelectronics progress. Vol. 423, 41 (2001).

[3] Jian quan yao. Nonlinear optical frequency conversion and laser tuning technology [M]. Science press. 1995.

[4]Yuan hong sun, the study of the theory of the nonlinear optical properties of organic materials

[D]. Shandong normal university, (2005).

[5] Jin Chen ,Jian quan yao ,Peng wang,et al.LD pumped all-solid-state frequency tripled ultraviolet laser [J].China Laser,2002,29(6):86-88.

[6] Yun wang,Xiu wei fan,Qian qian peng,Jie liu,Jinliang He. LD pumped Nd: GdVO4 three times the frequency ultraviolet laser crystal LBO. Optoelectronics, lasers. [J] in May 2005, 16 (5).

[7] Jiang yan, Shen wu xie,Xue lin yang. Three times the frequency of KDP crystal experiments and analysis. Journal of optics. [J]. Journal of October 10, 1998. 